\title{
Quel développement sans état ? Le cas d'Haïti
}

Résumé :

Nul pays n'est davantage qu'Haïti l'objet de la compassion et de l'aide internationale. Pourtant la population lutte pour survivre. À qui la faute ? À l'État, aux ONG, aux puissances étrangères, aux multinationales, aux élites locales?

Alice Corbet, anthropologue, spécialiste des camps de déplacés et de réfugiés et du dispositif humanitaire, mène des recherches en Haïti depuis 2011. Pierre Duquesne, ambassadeur chargé des questions économiques de reconstruction et de développement, a coordonné l'aide française en Haïti après le séisme de 2010. Jacqueline Plaisir, volontaire permanente d'ATD Quart Monde, originaire de la Guadeloupe, a vécu en Haïti de 2001 à novembre 2010, avant de rejoindre la délégation générale d'ATD Quart Monde international.

Le 12 janvier 2010, le séisme fait des dizaines de milliers de morts en Haïti (1). Est-ce uniquement le résultat d'une « catastrophe naturelle »?

Jacqueline Plaisir - Il est difficile de connaître le nombre exact de victimes : les chiffres du recensement sont imprécis car beaucoup n'avaient pas de papiers d'identité ni de toit pour vivre. Le séisme fut d'une grande magnitude, mais la vétusté, la manière dont l'agglomération s'est développée et l'absence de plan de secours ont aggravé le bilan: dix jours après, des vivants étaient encore sous les décombres, malgré les efforts incroyables de tous pour sauver des vies...

Ce séisme est survenu alors qu'Haïti traversait déjà une période difficile Après la chute de la dictature, à la fin des années 1980, une sorte d'euphorie a gagné le pays. Le plébiscite pour Jean-Bertrand Aristide en décembre 1990 (67\% des suffrages) s'est accompagné d'un véritable élan, avec le retour de la diaspora, le soutien international... Un formidable espoir aussitôt douché par le coup d'État (2). Le second mandat de ce président (2000-2004) se déroule dans la plus grande confusion. 
Des gangs se reconstituent dans des quartiers comme ceux situés sur les hauts de Martissant (Grande Ravine, Ti-Bois Descayettes...). Les manifestations contre le pouvoir, organisées par la société civile, dont les étudiants, s'amplifient. Le président est contraint au départ. Entre 2004 et 2006, les partisans du gouvernement déchu lancent l'opération « Bagdad», commettant de nombreux enlèvements. La période de désarmement, menée par la police et la Minustah (3) [Mission des Nations unies pour la stabilisation en Haïti] à partir de 2007, fut aussi très dure. Incitation à la délation, arrestations aveugles. Ce climat délétère a miné la confiance dans les quartiers. Les habitants des quartiers concernés ont tenté de rebâtir la confiance entre voisins, aspirant à la paix. Dans les hauts de Martissant, nous en avons été témoins. Une mère a refusé que l'on venge sa fille, tuée, pour ne pas perpétuer le cycle de la violence. Des jeunes ont cherché, à travers l'art ou des actions culturelles pour les petits, à « désarmer» les esprits. Les Haïtiens ont dû faire face, avec courage, à d'autres situations difficiles: les inondations meurtrières en 2004, la flambée des prix de produits de première nécessité en 2008 qui ont provoqué des manifestations, des « émeutes de la faim », quatre cyclones durant le seul mois de septembre 2009...

Pierre Duquesne - Ce terrible bilan n'est pas le résultat de la seule catastrophe naturelle. Quelques semaines plus tard, un séisme quasiment de même magnitude avait lieu à Valparaiso, au Chili et ne faisait Presque pas de morts. En Haïti comme ailleurs, l'état de nature renvoie à la nature de l'État: il n'y avait guère d'institutions étatiques. Autrement dit, pas de prévention des risques et de constructions antisismiques, pas de minis-tère du Logement (il n'y en a toujours pas aujourd'hui), pas de cadastre, pas de sécurité physique ni institutionnelle, assez peu d'organisation de la cohésion sociale, guère de gouvernance centrale, ni locale. La catastrophe a affecté la capitale assez uniformément, sans distinction de milieu social. Ce séisme a touché des quartiers comme Pétionville, où vivent les plus riches, jusque-là épargnés par les inondations. Pour la première fois, l'élite haïtienne a été touchée par une catastrophe naturelle dans sa chair et dans ses biens.

Alice Corbet - J'ai été impressionnée par le formidable niveau de cohésion sociale qui a vu le jour après le séisme. Le fait que riches comme pauvres aient été également touchés a créé une sorte de «communauté du malheur». Ce fut un événement fondateur de solidarités nouvelles, à travers la formation de comités et l'installation 
de la vie dans les camps. Mais les plus riches en sont vite sortis et les autorités ont manqué l'occasion de s'appuyer sur ces réseaux, car la majorité des camps a été éparpillée.

\section{L'aide internationale a été massive au lendemain du séisme. Quels visages a-t- elle pris ? À quels besoins répondait-elle ?}

J.Plaisir - L'aide d'urgence est parvenue très rapidement du monde entier, mais rien n'était prévu pour la canaliser. Dans des quartiers comme Grande Ravine, situé sur les flancs d'un morne (colline), beaucoup de gens ont refusé de partir pour rejoindre les camps, pour ne pas perdre une de leurs forces : pouvoir se soutenir entre voisins. Mais l'aide a été absorbée par les lieux les plus accessibles, les zones connectées par la route, sur terrain plat. Ceux qui habitaient dans les quartiers inaccessibles en voiture ont été atteints par les secours plus d'un mois après. Ce qui a été difficile, c'est que les Haïtiens n'ont guère pu participer aux discussions sur l'affectation de l'aide: souvent, les réunions étaient en anglais ! L'après séisme a suscité un grand espoir, celui d'une société sans classe. Une maman me disait : «On est tous ensemble dans la rue : il faudrait que la reconstruction permette à tous de retrouver un logement en même temps. Bientôt, nous serons les seuls encore à la rue. \

P. Duquesne - Il y a eu le scandale des réunions en anglais. Mais, globalement, les choses se sont mieux passées qu'on ne l'avait craint au départ. La France est intervenue rapidement grâce aux départements des Antilles. L'aide d'urgence en Haïti a fait l'objet d'une coordination sans précédent entre organisations internationales, États et acteurs non étatiques, intervenants civils et militaires. Les catastrophes annoncées (insécurité alimentaire, pandémies, poussées de violence...) n'ont pas eu lieu. Les Haïtiens ont fait preuve de beaucoup de courage et les camps, où l'eau était potable, ont joué un rôle important.

Certes, dans une telle situation, les bailleurs, publics comme privés, font face à une forte pression de la part des contribuables ou des donateurs, qui attendent des résultats rapides. Mais nous sommes parvenus à assurer un certain continuum entre 
aide d'urgence et développement, en partie grâce aux questions posées par la presse dès le lendemain de la catastrophe : quelles sont les racines de la crise ? Quelle est la pérennité de l'aide ? L'implication des Haïtiens aurait sans doute pu être meilleure, mais les Nations unies ont très tôt entrepris une large enquête dans les camps, «Une voix pour les sans voix », pour que les Haïtiens soient partie prenante du processus de reconstruction en préparation de la conférence de New York du 31 mars 2010. A. Corbet - L'inégalité dans l'approvisionnement humanitaire et les problèmes de coordination ont créé des tensions, sans toutefois conduire à l'explosion de violence annoncée. Si les Haïtiens ont survécu, c’est que beaucoup vivaient déjà auparavant de «la débrouille»: on ne sait pas le matin si on mangera le soir, donc on cherche un petit travail journalier. L'aide a permis d'enlever les débris, de remettre les routes en état, d'installer des infrastructures sanitaires, de fournir quelques solutions de relogement (dont des abris «provisoires»!), mais, dans certains lieux, elle est finalement repartie assez vite et la vie a repris son cours, dans une précarité souvent accrue par le désordre créé par le séisme.

J. Plaisir - Les gens ont continué à survivre comme avant. L'aide leur est apparue comme une manne ponctuelle, à laquelle ils n'ont pas toujours eu accès. Dans la consultation de l'Onu «La voix des sans voix », les membres du Mouvement ATD Quart monde vivant dans les quartiers du Haut Martissant insistaient sur le fait qu'il fallait partir de la situation des très pauvres, donner du travail à tout le monde. Or on a accordé moins d'attention aux lieux où les gens tentaient de s'en sortir par euxmêmes. Ils expri maient le souhait que le Palais national soit reconstruit par des ingénieurs et des architectes haïtiens, question de fierté... En vain (4).

\section{Quels sont les effets de cette intervention dans la durée ?}

P. Duquesne - Il a fallu deux ans aux États-Unis pour retirer les gravats du World Trade Center à New York, il n'en a pas fallu davantage en Haïti pour un volume cinq à dix fois supérieur (même s'ils n'ont pas été traités de la même manière). Le nombre de personnes dans les camps a considérablement diminué. Quant aux projets de développement, ils continuent: la France s'occupe par exemple de la reconstruction 
de deux quartiers, Baillergeau et Martissant. Ces opérations sont longues, car élaborées avec la participation des habitants.

Mais pour qui nourrissait l'espoir que cette catastrophe absolue serve de choc salvateur, c'est peut-être la déception. Au fond, on est revenu à la situation d'avant 2010 : les donateurs sont un peu mieux coordonnés, mais l'État est malheureusement aussi faible. On a pourtant tenté quelque chose d'inédit : la loi haïtienne a créé, pour dix-huit mois, une Commission intérimaire pour la reconstruction d'Haïti (CIRH) composée à parité d'Haïtiens (représentants de l'exécutif, du Parlement, du pouvoir judiciaire, des collectivités locales, de la société civile) et de représentants de bailleurs. Ce fut le lieu de vrais débats sur les stratégies et les projets. Mais quand cette Commission a cessé de fonctionner, nous n'avons pas vraiment su ce que voulaient les autorités haïtiennes. Elles n'avaient pas créé, dans l'intervalle, une agence de reconstruction comme elles disaient le souhaiter au départ.

A. Corbet - Certains quartiers s'en sortent mieux que d'autres : ceux où des acteurs de l'aide sont implantés de longue date, ou ceux où la cohésion était forte avant le séisme. Mais là où les ONG de développement ont souvent une bonne compréhension du contexte et des populations, certaines ONG d'urgence ont débarqué sans connaissance du terrain ni mémoire institutionnelle, avec un fort turnover, un personnel formé mais très jeune, et la pression de dépenser vite pour répondre aux demandes des bailleurs, souvent au profit des femmes, de la « résilience », etc., autant de mots-clés d'allocation de l'aide. Ces interventions parfois redondantes ont abouti à des détournements, par exemple en instituant comme chefs de certains quartiers des nouveaux venus parce qu'ils parlaient un peu anglais. Ces échecs ont été facilement attribués à de «mauvais bénéficiaires »! D’autres quartiers méconnus ou nouveaux ont, quant à eux, été oubliés par les organismes de solidarité comme par l'État haïtien. Enfin, une part non négligeable de la population est ignore par tous : les plus pauvres, qui ne se sentent pas la légitimité de réclamer quelque chose, voire d'exister. Ces gens sont donc demeurés invisibles après le séisme, réduits à la plus grande précarité, malgré l'intervention humanitaire massive.

Or, autant dans l'urgence, avec un État défaillant appelant au secours, la légitimité de l'aide ne souffre guère de contestation, autant dans la durée, les interventions étrangères risquent de se substituer à l'État. Elles vont parfois de pair avec 
l'affaiblissement des services publics. Elles captent la matière grise restée au pays au détriment des institutions nationales (qui paient moins bien). Ainsi, le paysage de l'aide se caractérise par une surenchère de sigles, de comités, de commissions, qui continuent de se créer en parallèle des structures publiques existantes. Quelle en sera la pérennité ? Ce mouvement contribue à déresponsabiliser l'État haïtien, comme si au bout d'un moment, l'humanitaire se justifiait à lui-même, par lui-même.

Mais si l'aide se substitue à l'État, c'est aussi faute de relais. En effet, la société haïtienne est très dilatée : une élite très petite et individualiste possède la majorité des richesses du pays (entreprises, terres, etc.), et cherche surtout à tirer son épingle du jeu, quel que soit le gouvernement en place. Et la désorganisation générale n'a pas aidé à ce que des fonds attribués soient bien répartis et distribués...

J. Plaisir - Les Haïtiens attendent qu'on les soutienne dans leurs efforts pour donner corps à leur aspiration : une société qui se bâtisse sans que l'un prenne le pouvoir sur l'autre. La société est inégalitaire, mais elle recèle aussi beaucoup de forces de solidarité : «Les voisins, c'est drap blanc», «Les voisins, c'est la famille». Qui descendra une personne malade à 2 heures du matin pour se faire soigner, sinon un voisin ? J'ai vu une femme sortir cinq gourdes [monnaie locale] cachée dans sa chevelure épaisse pour les donner à sa fille : «Tu te débrouilleras avec ça! » En Haïti, on n'accumule pas, on donne, on partage. Demain, on recevra à son tour... Les Haïtiens ont été choqués de voir la distribution d'aide escortée par des militaires : « Nous ne sommes pas des voleurs ! » Là où des jeunes l'ont assurée, ce fut sans heurts, nous l'avons expérimenté. Beaucoup se mettent debout pour reconstruire, sans faire de bruit. Mais pour quelques projets (comme le cash for work) qui s'appuient sur des initiatives de base, combien sont pensés de l'extérieur et démobilisent les forces locales ? Je me rappelle ce projet d'unité obstétrique qui devait renforcer la Clinique partenaire de notre projet santé ; après moult négociations, l'ONG a choisi de monter sa propre structure, embauchant des matrones (sages-femmes) formées par la clinique locale. Ces professionnels devenaient ainsi indisponibles pour les accouchements à domicile qu'elles assuraient depuis des années pour les familles les plus fragiles et les plus isolées. Il n'est pas évident de prendre en compte les plus pauvres dans les projets. Il leur est difficile d'oser exprimer leur point de vue. Souvent, ils vivent dans l'angoisse que les projets se traduisent par de nouveaux 
déplacements de populations, ou par des divisions. Il faut du temps pour créer la confiance et la connaissance, alors que les responsables de projet, venus d'ailleurs, changent trop vite, parfois tous les trois mois, et que les bailleurs ont besoin de décaisser rapidement les fonds, sans faire d'évaluation deux ou trois ans après.

\section{Vous avez parlé d'État absent ou défaillant, d'autres parlent d'État failli. Que recouvre ce diagnostic?}

A. Corbet - De manière générale, on décrit un «État failli» selon les critères suivants : un gouvernement central faible sur tout le territoire, l'absence de services publics, une corruption généralisée, l'absence d'État de droit, une instabilité et des déplacements de populations, une crise économique. Haïti remplit tous les critères ! Les Haïtiens aspirent à une gestion du bien commun, à un État fort capable d'assurer la sécurité, du travail, un système éducatif et sanitaire cohérent... Mais il manque une structuration démocratique de base : quel intérêt pour les gens d'avoir des papiers d'identité s'ils n'ont pas confiance en l'État ou s'ils pensent que ce dernier ne peut rien leur apporter de bon ? Si l'État ne sert à rien, à quoi bon s'impliquer dans la vie nationale ou en attendre quelque chose?

P. Duquesne - Pour qualifier un État de «failli », il faut d'abord qu'il y ait un État. Deux critères me paraissent significatifs. Le taux de prélèvements obligatoires était en Haïti d'environ $11 \%$ du Pib, soit quatre à cinq points au-dessous de la moyenne de l'Afrique subsaharienne. Ce taux traduit la capacité politique et technique des pays à collecter l'impôt et leur volonté de redistribution. Sans recettes fiscales suffisantes, comment assurer les coûts de fonctionnement des projets financés par l'aide internationale?

Un second critère est la volonté politique des élites à décider et à mettre en œuvre. Il est trop facile, comme Raoul Peck dans son film Assistance mortelle, de rejeter la faute sur les bailleurs. Une vision de long terme était énoncée par les Haïtiens euxmêmes dès la conférence des donateurs en mars 2010 à New York. Mais l'occasion n'a finalement pas été saisie. On déplore la noria des ONG et des bailleurs, mais les ministres haïtiens n'ont malheureusement cessé de changer depuis quatre ans dans 
tous les domaines clés. La Constitution de 1987, par crainte de la dictature, a multiplié les contre-pouvoirs, au point de rendre le pays parfois assez difficile à gouverner.

J. Plaisir - Haïti était, lors de l'indépendance en 1804, la perle des Antilles. Les anciens esclaves ont mené la lutte pour la liberté et conquis l'indépendance grâce à leur alliance avec la classe des affranchis et des mulâtres. Deux visions coexistaient : celle des anciens esclaves, craignant toute forme de domination ou d'exploitation, désireux de préserver la liberté et de créer une société égalitaire. De la part des autres, la volonté de maintenir le système hérité de la colonie avec un État, une organisation institutionnelle. Ces deux visions ont tracé deux projets parallèles, avec peu d'interférence. En outre, la nation naissante a dû payer très cher son indépendance en indemnisant les planteurs et colons français pendant près d'un siècle ! Depuis, la situation est figée. L'État fait l'objet d'un divorce avec la société : on refuse de le créer vraiment tant que l'on n'est pas sûr qu'il agisse pour le bien commun. Le fossé ne se comble pas. Les élites ont la plus grande difficulté à aller à la rencontre de tous ceux qui aspirant à une société plus égalitaire. L'éducation est à deux vitesses. L'université elle-même, destinée à former les élites, est en souffrance, alors qu'il y a une grande soif d'éducation.

Vous faites le procès des élites. Mais l'intellectuel haïtien Lyonel Trouillot, lui, voit son pays «sous occupation»: «Penser l'avenir d'Haïti, c'est penser sa désoccupation, sa dés-ONG-isation (5) ».

A. Corbet - Les murs d'Haïti parlent : «ONG occupation», «Blancs (6) dehors ». Ces inscriptions traduisent un discours très prégnant, même si cette accusation est assez injuste. Certes, les conditions de vie des ONG sont très éloignées de celles des Haïtiens : les expatriés habitent généralement «haut la ville», dans les mêmes quartiers que les élites. Rares sont ceux qui parlent créole. Le 4x4 blanc avec un sigle est devenu le symbole de cette présence. Or, quand les projets réduisent les «bénéficiaires » à la passivité, ceux-là se servent de leurs deux mains : l'une pour réclamer, l'autre pour frapper quand ils jugent l'aide mal ciblée. 
Les murs sont aussi couverts du slogan «ONG=ONU= choléra $»(7)$. Qu'une structure censée aider importe ce nouveau fléau a marqué les esprits, d'autant que le choléra a touché des zones reculées. De ces amalgames et incompréhensions, il résulte une certaine colère: des $4 \times 4$ se font taper dessus. Quand, de plus, les occupants du véhicule ont à peine 20 ans et aucune connaissance du terrain, cela renvoie une image très négative et contribue à nourrir le sentiment de vivre sous occupation. Mais il s'agit bien d'une image.

P. Duquesne - La vision de Lyonel Trouillot est assez partagée en Haïti. La méfiance est telle que même la diaspora est perçue comme un potentiel danger, alors qu'elle a une volonté réelle d'aider son pays et qu'elle contribue par ses transferts à 10-15\% du Pib (un argent non investi, mais consommé). Dans le même temps, Haïti est un pays qui attire légitimement la compassion. Beaucoup d'ONG y interviennent, avec les meilleures intentions du monde, parfois au-delà de leur mandat. Mais il est vrai qu'elles peuvent contribuer, par leurs salaires et leurs modes d'action, à instaurer une logique de marché dans des secteurs qui pourraient être publics.

Les Haïtiens se donnent-ils vraiment les moyens de se substituer aux bailleurs ? L'alternative est simple. L'aide internationale va se faire plus sélective. Que diraient les mêmes intellectuels haïtiens si les États et les ONG cessaient leur aide ? Sûrement crieraient-ils à l'abandon. Lors d'une conférence, en juin 2010, le président René Préval rappelait: «Il y a cinquante ans, nous avions le même niveau de revenu par tête que la République dominicaine.» Il regrettait que la situation économique ou institutionnelle actuelle soit le résultat de l'action des Haïtiens eux-mêmes.

J. Plaisir - L'aide internationale transite beaucoup par les ONG, ce qui leur confère une vraie influence. Or «la main qui donne est au-dessus de celle qui reçoit » : une posture qui met d'emblée les acteurs locaux en position d'infériorité, à moins que les ONG ne s'engagent avec eux dans un partenariat durable. Le principe de l'aide n'est pas en cause, mais les gens concernés ont le droit de dire ce qui est bon pour eux. Eux seuls ont la mémoire du territoire, eux seuls savent le prix à payer pour des projets mal conçus. Ils ne parleraient pas d'occupation s'ils avaient prise sur ces interventions étrangères. Dès lors que l'on travaille ensemble, les initiatives peuvent fonctionner, nous en faisons l'expérience. Ce travail sur un pied d'égalité est freiné 
par de fortes inégalités de statut entre les habitants, les fonctionnaires et le staff d'ONG qui gagnent près de 1000 euros par mois (une fortune en Haïti !).

Propos recueillis par Jean Merckaert.

\section{Notes :}

1. Entre 46000 et 85000 morts selon un rapport de l'agence américaine d'aide au développement (USAID), de 200000 à 300000 selon le gouvernement haïtien [NDLR].

2. La junte sera renversée en 1994 par l'armée américaine [NDLR].

3. La Minustah était présente en Haïti depuis le conflit armé de 2004 [NDLR].

4. Le président Martelly a confié la reconstruction du Palais national à J/P Haitian Relief Organization, ONG de l'acteur Sean Penn [NDLR].

5. «On ne peut pas pactiser avec la catastrophe». Entretien accordé à Faim et Développement Magazine, sept.oct. 2013, p. 9.

6. En créole, «blanc(s)» signifie « étranger(s)».

7. L'émergence du choléra en novembre 2010 était sans doute imputable aux soldats de la Minustah dans la région de l'Altibonista. 